\title{
Die Herkunft der Ungarn - Tradition und Mythologie der orientalischen Abstammung
}

\author{
TIBORC FAZEKAS (Hamburg)
}

\section{Vorbemerkungen}

Erinnern und Vergessen sind zwei, meistens spontane und komplementäre Vorgänge in der menschlichen mentalen Aktivität, die im Leben des Individuums die Funktionsfähigkeit des Denkens ermöglichen. Sie stehen in einem komplizierten Verhältnis zu einander, können gesteuert werden, aber darin besteht bereits die Möglichkeit ihrer Manipulation und ihrer Umwandlung in Obsession und Verdrängen. Auch im Leben der organisierten menschlichen Gesellschaften spielen die Vorgänge des kollektiven Erinnerns und Vergessens eine gestaltende, tragende Rolle, allerdings nicht mehr im spontanen Zusammenhang. Zur Lenkung des kollektiven Erinnerns und des Vergessens sind im Laufe der Jahrtausende der menschlichen Kulturen viele Methoden und Mittel entstanden; Riten, Symbole, Mythen, Glaubenskonstruktionen, Zeremonien, Künste, Ideologien sind die bekanntesten Fassungen und Formen ihrer Artikulation. Die Erklärungen für die Abstammung, die Herkunft und für die Zukunft der einzelnen Menschengruppen sind charakteristische Gebiete, die traditionell von irrationalen Elementen bestimmt oder zumindest durchwoben sind. Diese in der Folklorekunde als Herkunftsmythen oder Herkunftssagen definierte Gruppe mündlich tradierter Texte ist immer ein fester Bestand der kulturellen Identität gewesen. Die Frage über die Inhalte oder gar über die Richtigkeit, die Glaubwürdigkeit dieser Texte konnte nicht einmal gestellt werden, denn viele von ihnen haben im Laufe der Zeit einen zusätzlichen rituellen oder sakralen Charakter erhalten. Erst in den letzten drei Jahrhunderten, als die traditionell vom Glauben und von mythologischen Traditionen bestimmten Gebiete der menschlichen Kultur in der Wissenschaft einen „Konkurrenten“ bezüglich der Sinnesfrage der menschlichen Existenz erhalten haben, wurden die Traditionen mit modernen Erkenntnissen konfrontiert. Die wissenschaftlichen Untersuchungen haben im Vergleich zu den Mythen oft ganz andere Gründe und Zusammenhänge zu Tage gefördert, haben vieles zu erklären, tradierte Vorstellungen zu rationalisieren, zu korrigieren, eine Alternative zu diesen Texten anzubieten versucht. Diese rational ausgerichtete Entmythologisierung hat aber in sehr vielen Fällen zum starken gesellschaftsinternen Widerstand, nicht selten sogar zu internationalen oder eher interkultu- 
rellen Spannungen geführt. Sie berührt nämlich die individuelle oder gruppenbezogene Identität des Menschen.

Einerseits sind zumindest Elemente dieser Mythen teilweise kulturell sehr tief in der Psyche des Individuums, in seinem Unbewussten verankert, ihre „Entblößung“, ihre Korrektur oder rationale Widerlegung würde der Psyche bereits auf individueller Ebene tragende Komponenten rauben, ohne einen psychologisch oder spirituell vergleichbaren wirksamen Ersatz dafür anzubieten (,interne Gefährdung“). Andererseits sind zahlreiche Elemente und Motive, manchmal sogar objektive geographische Gegebenheiten der Mythen von menschlichen Gesellschaften in mehreren Kulturen parallel vorhanden, eine auch wohlgemeinte Gegenüberstellung dieser Tatsachen kann wiederum die jeweiligen menschlichen Gruppen (Nationen oder Kulturen) allzu leicht gegeneinander aufhetzen (,externe Gefährdung“").

Die Geschichte der Vorstellungen der Ungarn über ihre Herkunft entspricht dem oben skizzierten Schema fast lückenlos. Der Konflikt zwischen der Überlieferung und den wissenschaftlichen Feststellungen ist vor etwas mehr als zweihundert Jahren ausgebrochen und trotz des Konsenses unter Wissenschaftlern, von der breiteren, nicht-fachlichen Öffentlichkeit bis heute nicht für beendet erklärt. In Anbetracht der Komplexität dieser Phänomene sowie wegen der widersprüchlichen und intensiven, in der einschlägigen Fachliteratur Hunderte von Büchern und Tausende von Aufsätzen hinterlassenden Diskussionen um dieses Thema, kann ich mich bei den folgenden Ausführungen nur auf manche charakteristische, offensichtlich mit der Wissenschafts- und Kulturgeschichte der Ungarn zusammenhängende soziologische und mentale Komponenten konzentrieren.

Vor allem möchte ich betonen, dass in Bezug auf die zentrale Frage meines Beitrags, in Bezug auf die Herkunft der Ungarn, ebenso, wie bei anderen Völker, wir uns auf keine alles erklärende Tatsache oder einen Beweis berufen können.

Die Geschichte der Menschheit und ihrer Teilkulturen ist nur bis zu einer bestimmten Zeit zurückzuverfolgen, die Vor- und Frühgeschichte ist aus der gemeinsamen Erinnerung verschwunden und kann deshalb nicht lückenlos ermittelt werden. Wir wissen, dass das Volk der Ungarn nicht dort gelebt hat, wo es sich heute befindet, aber das ist keine besondere Eigenschaft dieses Volkes. Die historischen Quellen setzen die Besiedlung des heutigen von Ungarn bewohnten Kerngebietes etwa auf das Jahr 895 n. Chr. (in der ungarischen Geschichtsschreibung heißt dieses Ereignis „Landnahme“). Aus anderen Quellen gibt es zahlreiche weitere Beweise dafür, dass die später als Ungarn benannten Völkergruppen aus dem Osten gekommen sind. ${ }^{1}$ Aber über diese sporadischen Angaben, Beschreibungen und Hinweise hinaus haben wir keine als objektiv zu bezeichnenden Informationen über die Vergangenheit der Ungarn mehr. Bei der Klärung der Frage wird dadurch der Phantasie und somit

1 Kovács / Veszprémy 1996. 
allerlei Konstruktionen eine entscheidende Rolle zugeteilt. Es stehen zwar verschiedene Angaben, Nachrichten, Funde und Materialien für einschlägige Untersuchungen zur Verfügung, aber sie sind allesamt fragmentarisch und können keinen absoluten Beweis zur Klärung der Herkunftsfrage erbringen. Wir haben es infolge der geschilderten Lage also mit unterschiedlichen Konstruktionen zu tun, und diese können einander ergänzen oder stärken, schwächen oder widerlegen, ein spekulatives Element bleibt in ihnen immer erhalten. Zu Recht kann (allerdings nicht ausschließlich in Bezug auf die Ungarn!) festgestellt werden:

Die Ursprünge der Ungarn bleiben bislang für die moderne Forschung noch weitgehend im Dunkeln verborgen. Die teils beachtlichen Ergebnisse von Archäologen, Anthropologen, Sprachwissenschaftlern, Ethnologen und Historikern sind, bei allen Bemühungen, noch Stückwerk, das nur Schemen der ungarischen Frühgeschichte erahnen lässt. ${ }^{2}$

Natürlich gab es in Bezug auf die Bewertung der historisch jeweils vorhandenen Kenntnisfragmente und auch in Bezug auf die Methoden ihrer Aufarbeitung eine deutliche Entwicklung im Laufe der Geschichte, aber noch heute spielt die eigene Überzeugung, die wissenschaftliche Einstellung, die Zugehörigkeit des Forschers zu irgendeiner Richtung, Werkstatt oder Tradition und eine - zumeist wunschgesteuerte - Vorstellung des Publikums bezüglich der Aussagen eine soziologisch relevante Rolle bei den Untersuchungen zur Urgeschichte der Ungarn.

Diese seit mehreren Jahrhunderten andauernden Diskussionen und die vorgebrachten Argumente kann ich natürlich im Rahmen einer kurzen Darstellung nicht lückenlos präsentieren. Ich möchte mich im Folgenden deshalb wesentlich auf zwei Typen des Umgangs mit den bereits erwähnten Fragmenten konzentrieren. Den ersten würde ich als „faktenflickenden“, den anderen eher als „fiktiv-literarischen“ bezeichnen. Auf die erste Methode sind zahlreiche wissenschaftliche Disziplinen eingegangen, ihre Ergebnisse werde ich ebenso kurz schildern, wie die Ergebnisse einer durch die Tätigkeit von kreativen Menschengruppen und Individuen im Laufe der Jahrhunderte entstandenen reichhaltigen, an Texte gebundenen (literarischen) Tradition der ungarischen Kultur in Bezug auf die Herkunft des ungarischen Volkes. Dass sich dabei zahlreiche Widersprüche und Konflikte ergeben haben und diese einstigen Reibungen auch die heutige Situation weiterhin mitprägen, ergibt sich als selbstverständlich angesichts der Komplexität des Phänomens. Deshalb und wegen der Komponenten der Irrationalität in den Überzeugungen wird es unvermeidlich sein, auch gewisse ideologische und politische Relevanzen bei den verschiedenen Vorstellungen zu berücksichtigen.

2 Kellner 1997, S. 76. 


\section{Historische und sprachwissenschaftliche Theorien bzw. Rekonstruktionen}

Zuerst möchte ich deutlich auf die historische Komponente in der Entstehung eines gemeinschaftsbezogenen Bewusstseins bei den Ungarn hinweisen. Als am Ende des 9. Jahrhunderts der ungarische Stammesverband das Karpatenbecken dauerhaft besetzt und in den folgenden Jahrzehnten zahlreiche Raubzüge in unterschiedlichen Regionen Europas durchgeführt hat, kümmerten sich die beteiligten Menschen wohl kaum um eine Legitimierung ihres Handelns. Einerseits hatten sie ihre gefürchteten Schießbögen, ihre schnellen Pferde und meistens auch irgendeinen politischen Verbündeten oder zumindest Anstifter hinter sich, die neben der erhofften Kriegsbeute ihr Verhalten nach innen wie nach außen genügend begründet haben. Andererseits war ihre Organisationsstruktur, der Verband von Stämmen, dafür verantwortlich, dass sie ihre Existenz und ihr Handeln mit Betonung der von den frühfeudalen europäischen Strukturen abweichenden Inhalte und Formen ihres Lebens definierten. Ihre Ansichten basierten eher auf symbolischen Vorstellungen und auf zeitlich unmittelbar vorausgegangenen Handlungen, eher auf der Vorstellung (und Realität) der Zusammengehörigkeit einer überschaubaren Menschengruppe, des Stammes (etwa 3-4 Generationen der unmittelbaren Blutsverwandten), und sie hatten vor allem nicht die zeitlich ausgedehnte, durch Erbfolge der führenden Persönlichkeiten (oder eines Geschlechts) gesicherte diachrone Vorstellung einer größeren Entität, einer Nation. Die Ursachen für diese flexible, sich rasch ändernde Identität (die sich unter anderem auch in den verwirrenden Variationen der externen und internen Benennungen der betroffenen Völker verfolgen lässt) und für die Mentalität, die zu dieser Zeit noch keinesfalls die Annahme eines Bewusstseins auf der Ebene einer den damaligen westeuropäischen Begriffen entsprechenden Nation zulässt, haben ihre Gründe in der Vorgeschichte der Ungarn, als sie selbst in den Steppenregionen Europas Jahrhunderte lang in den sich oft rasch ändernden Stammesverbänden von verschiedenen Turkvölkern integriert waren und meistens als eine von zahlreichen untergeordneten Gruppen lebten.

Die Ungarn (aber nicht nur sie) scheinen im Laufe eines imaginären $\mathrm{Zu}$ rückspulens der Geschichte zu verschwinden, ihre Spur wird immer unsicherer, und die überlieferten Angaben über sie werden gleich von mehreren anderen heutigen Völkern als ihre Hinweise auf ihre eigene Vergangenheit beansprucht. Wir wissen, dass die Ungarn sich selbst als „,magyar“ bezeichnen, aber dieser Name erscheint in den historischen Quellen erst recht spät, zum ersten Mal können wir darüber bei Anonymus am Ende des 12. Jahrhunderts lesen:

per ydioma aliegnigarum hungarii et in sua lingua propria mogerii vocantur. ${ }^{3}$

3 Silagi 1991, S. 29. 
In der nicht ungarischsprachigen Textüberlieferung bezeichnete man sie unter anderen Namen, welche aber nicht exklusiv für die heutigen „Magyaren“ gegolten haben. Die byzantinischen Autoren haben gerne die Bezeichnung „,turkoi“ (auch) für die Ungarn benutzt, der (wohl die Zustände des Stammesverbandes vor der Landnahme korrekt wiedergebende) Name „onogur“ (d.h. „,zehn Oguren“) ist hauptsächlich in den slawischen Quellen als „,ungri“ erhalten geblieben und diente als Grundlage für die heute am weitesten verbreitete fremde Bezeichnung der Ungarn (,,ungarus/hungarus“). Aber die historisch relevanten Quellen haben die Vorfahren der heutigen Ungarn auch mit den Namen „sawartoi asphaloi“; „,baschkiren“; „madschar“ (hauptsächlich in den persisch-arabischen Texten) bezeichnet, aber auch die Skythen, Hunnen, Awaren, Parther sind bei der Suche nach möglichen frühen Benennungen ebenso herangezogen worden wie die Sarmaten oder Jazygen. All diese Namen wurden in der Zeit vor und um die Landnahme herum in den europäischen Aufzeichnungen so aufgenommen, dass dabei - wohl in Folge der seit längerem stattfindenden Völkerwanderung - sowohl offensichtliche Verwechslungen mit anderen ,barbarischen“ Völkern (unter anderen mit den Sarazenen) vorliegen, als auch die kumulative Übertragung von seit langem überlieferten Feindbildern und -Motiven ohne nähere Betrachtung des „Konkreten“ eine Rolle gespielt haben dürften. ${ }^{4}$

Mit den deutlichen Änderungen der allgemeinen Bedingungen durch die Landnahme, nachdem der ungarische Stammesverband an die westlichste Stelle der nomadisierenden Turkvölker und somit in die unmittelbare Nähe der im Westen Europas sich etablierenden feudalen Staatsstrukturen gelangt war, stellte sich jedoch durch die veränderten politischen und militärischen Kräfteverhältnisse des 10. Jahrhunderts immer deutlicher die Aufgabe, mit der geopolitischen Umgebung statt Konfrontation auf Kooperation zu bauen, um das Behalten der besetzten Region zu sichern. Diese Erkenntnis führte durch Anpassung zu zwei gravierenden, entscheidenden Veränderungen im Selbstverständnis der Ungarn für die Zukunft: Sie mussten einerseits ihren alten Vorstellungen über die Welt und sich selbst abschwören, ihre mentale Identität (hauptsächlich vertreten in ihren Glaubensvorstellungen), ,das Heidentum“, zu Gunsten des christlichen Glaubens aufgeben, damit sie andererseits die zweite grundlegende Veränderung, die Gründung eines nach westeuropäischen Vorstellungen errichteten (auf festgelegte Erbfolge der führenden Schichten und dynastischen Verbindungen basierenden) feudalen Staates vollziehen konnten. Nach einer innenpolitisch konfliktträchtigen Zeit von mehreren Jahrzehnten konnte, nahezu zeitgleich mit anderen sich formierenden Nationen der Region (Polen, Tschechen), im Jahre 1000 das Königreich der Ungarn gegründet werden. Durch diese radikale und nicht gewaltlose Umstellung ist jedoch strukturell in den Köpfen eine nachhaltige Kluft, ein Konflikt zwischen althergebrachtem ,Eigenem“ und aufgezwungenem „Fremdem“ entstanden, welche

4 Róna-Tas 1999, S. 271-313. 
bis zu unseren Tagen hin die Verhaltensmuster und die Vorstellungen der Menschen und ihrer Kultur bestimmt haben. Je nach geschichtlicher Lage haben diese beiden psychologischen Grundeinstellungen einen abwechselungsreichen Kampf miteinander geführt. Die als „eigene“ bezeichnete Tradition der Ungarn ist im Laufe der Zeit „versunken“, sie wurde unter den neuen Bedingungen teilweise verfolgt, teilweise verboten, sie änderte ihre Form und hat vor allem ihre Vollständigkeit verloren. An Stelle eines komplexen Systems sind Reststücke davon in sehr unterschiedlichen Kontexten, Gattungen und Formen zerstreut erhalten geblieben, die wiederum nur mit Hilfe von Rekonstruktionen erkannt und verstanden werden können.

Als die Elemente der ursprünglich mündlichen Tradition sich im Laufe der Zeit zunehmend gegen eine sich langsam herausgestaltende, methodologisch geprägte wissenschaftliche Erforschung behaupten mussten, wurde dieser Konflikt zwischen dem „Alten“, Bewährten und „Neuem“ neu angefacht. Die oft über den Interessen der einzelnen Nationen stehenden, internationalen Merkmale der wissenschaftlichen Arbeit erhielten beim Laienpublikum beinahe automatisch einen feindlichen, ,antinationalen“ Charakter. Die kühnen, nach Objektivität strebenden Theorien der Wissenschaft stehen nämlich fast immer automatisch im krassen Gegensatz zu den romantischen, bunten und sich selbst gegenüber meist positiv gesinnten Vorstellungen der weitgehend statischen, auf das Wiederholen des Überlieferten sich stützenden Theorien der gegnerischen Seite.

In Bezug auf die Abstammung und Frühgeschichte der Ungarn verfügen wir, wie bereits gesagt, über keine handfesten Beweise, unwiderlegbaren Zeugnisse und eindeutigen Aussagen. Wie bei den meisten Völkern, musste auch hier eine glaubwürdige Herkunft erst rekonstruiert werden. $\mathrm{Zu}$ dieser Rekonstruktion stehen in ihrer Qualität und Quantität sehr unterschiedlich beweiskräftige Materialien zur Verfügung, die unter Anwendung von geeigneten Methoden zur Vermehrung des aktuellen Wissens beitragen können, sie werden jedoch nie die Gesamtheit der Fragenkomplexe um den Ursprung der Ungarn beantworten können.

Anders als bei den Finnen, wo das Kalevala, das Nationalepos, noch am Anfang des 19. Jahrhunderts einen fast kohärenten Entstehungsmythos der Welt, und darin der Finnen, für die zukünftigen Generationen überliefern konnte, sind bei den Ungarn die Spuren einer solchen epischen Tradition nicht nur in der Neuzeit nicht vorhanden, auch die früheren historischen Quellen können nicht auf einen solchen, inzwischen eventuell verloren gegangenen Stoff hinweisen, geschweige denn ihn zitieren. Diese Tatsache mag wohl wieder mit der früheren Stammesstruktur der Ungarn zusammenhängen: Hinweise zur Herkunft der einzelnen Stämme, totemistische Elemente, Mythologeme, fragmentarisch erhaltene Motive in der epischen Folklore haben sehr vieles aufbewahrt, aber diese Ansätze in der mündlich überlieferten Geschichte wurden zeitlich erst recht spät, wohl nach dem 10. Jahrhundert, zur 
Legitimation der Macht des führenden Stammes von Árpád zu einer „Geschichte der Magyaren“ (des gesamten Stammesverbandes) zusammengefügt.

Die uns so überlieferten Informationen sind natürlich keine wissenschaftlichen Erkenntnisse gewesen, sie waren lediglich Teile eines ,,vorwissenschaftlichen" Diskurses zur Erstellung einer Tradition und eines historischen Bewusstseins. Als in den folgenden Jahrhunderten die Anforderungen der Wissenschaftlichkeit zunehmend artikuliert und entwickelt wurden, gelangten die Elemente dieses Stoffes in die ,schöpferischen“ Bereiche der menschlichen Kultur, sie können heute der Korrektheit halber nur als epische Kunstwerke betrachtet werden. Die Problematik der gesamten Diskussion um diese Theorien ist eben hier zu verstehen: die früheren Annahmen, die tradierten Vorstellungen können zwar durch die immer neuen Erkenntnisse der Wissenschaft widerlegt werden, aber sie berühren die Phantasie erweckende Natur der Texte nicht und können ihre ursprünglich eminent wichtige psychologische und gesellschaftliche Funktion nicht unmittelbar ersetzen. Klar ist lediglich geworden, dass die Motive und Behauptungen des historisch herausgebildeten Stoffes an sich (ohne weiteres Beweismaterial) nicht dazu taugen, als Argument in einer fachgerechten wissenschaftlichen Diskussion eingesetzt zu werden. Die Lage ist mit der (schwer vorstellbaren) Situation vergleichbar, als wenn etwa jemand heute in Frankreich die literarisch überlieferte trojanische Herkunft der Franzosen als wissenschaftliche Erkenntnis in die Argumentation bei einem Diskurs einzubringen versuchen würde.

Im Laufe des Klärungsprozesses der ungarischen Ethnogenese standen die Annahmen und Rekonstruktionsversuche immer direkt einander gegenüber, die Entwicklung von wissenschaftlichen Forschungsmitteln hat allerdings zunehmend eine vor allem methodologisch ,standfeste“ Beweisführung verlangt, die die traditionellen Sagen, Legenden und Märchen sowie die literarischen Texte und mythologischen Theorien nur zunehmend schwer haben erbringen können. Deshalb ist heute kein Dialog mehr zwischen den Vertretern der verschiedenen Ansichten möglich, der wissenschaftliche Diskurs schließt viele von ihm abweichende Vorstellungen hauptsächlich wegen problematischer methodologischer Bestandteile aus, die Vertreter der nicht innerhalb des Wissenschaftsbetriebs erarbeiteten Theorien werfen ihrerseits wiederum den übrigen „,institutionalisierten“ Wissenschaftlern und somit der Wissenschaft schlicht den Verrat an den Interessen der Nation vor.

Die „,vorwissenschaftliche“ Phase der aufgestellten Herkunftstheorien beginnt mit der Zeit nach der Staatsgründung (1000), als die herrschende Dynastie, das Haus Árpád, vor allem nach außen eine europaweit akzeptierte Legitimierung ihres Machtanspruches erzielen wollte (nach innen legitimierten sich die Herrscher durchaus erfolgreich mit den Mitteln eines Feudalstaates). Die ersten eigenen Angaben über die Herkunft der Ungarn stammen natürlich aus der Zeit, als das Volk und das Reich der Ungarn seine Position in Europa festigen, begründen wollten. Die nationale Geschichtsschreibung beginnt mit den ersten Chroniken, in welchen bereits eine Darstellung (Konstruktion) der 
Urgeschichte und Herkunft der Ungarn geschildert wird. Da später diese Behauptungen auch in die international als Quellen angesehenen historischen Werken übernommen wurden, ist mit ihrer Hilfe eine „epische“ Tradition entstanden, die einerseits fortan lebt, andererseits von den verschiedenen inzwischen entstandenen Disziplinen andauernd kritisch untersucht wird. Hauptelement dieser Tradition ist die Anbindung der Ungarn an die Hunnen durch Verwandtschaft und der Rechtsanspruch des ungarischen Königs auf das einstige Reich Attilas. Die Urquelle dieser Auffassung sind die Gesta Hungarorum des anonymen Notars (Anonymus) von Béla III, der sein Werk - wohl unter Anwendung der Texte der inzwischen verloren gegangenen früheren „Urgesta“ nach 1196 verfasst hat. Die Idee einer Verbindung zu den Hunnen musste jedoch bereits früher existiert haben. Um 1063, also mehr als ein Jahrhundert vor den Gesta des Anonymus schenkte die Witwe von Béla I an Otto von Nordheim ein wohl im 10. Jahrhundert hergestelltes Schwert, das als „Schwert Attilas" bezeichnet wird, da Otto ihren Sohn Salomon im Kampf um den Thron unterstützt hatte. ${ }^{5}$ Diese Tatsache weist handfest auf eine Überlieferung der Verwandtschaft mit den Hunnen hin, die möglicherweise mit Hilfe der in Pannonien sesshaften slawischen Bevölkerungsgruppen tradiert wurde. Wieweit die Reste einer auf demselben Gebiet lebenden, jedoch zu dieser Zeit weitgehend assimiliert erscheinenden awarischen Bevölkerung ihrerseits diese Überlieferung mitgestaltet haben konnte, wurde wenig erforscht. Die Awaren, ein nomadisches Turkvolk, sind in ihrer Geschichte deutlich den Spuren der Hunnen gefolgt, auch ihr Schicksal, das „Verschwinden“ von der Bildfläche der Geschichte, haben sie geteilt. Sie könnten als tatsächliches Bindeglied zwischen den Vorgängern und den Nachkommen, also der Hunnen und der Ungarn in Pannonien gewirkt und eine - sonst nicht vorhandene - Gemeinschaft zwischen diesen drei Völkern mit hergestellt haben. Diese neue Tradition hat später wahrscheinlich durch die starke deutsche Präsenz am königlichen Hof zur Zeit der Herrschaft Istváns I (1000-1038) aus einer ganz anderen Richtung, aus der Hunnenkomponente des Nibelungenliedes, zusätzlich Unterstützung erhalten.

Zur Begründung des Machtanspruchs des ungarischen Herrschergeschlechts nach außen mit Hilfe der europäischen Traditionsmuster standen zu dieser Zeit allerdings nur recht eingeschränkte Wahlmöglichkeiten zwischen der Bibel, der Antike und den bekannten Größen der „Anti-Antike“ (Skythen, Hunnen, etc.) zur Verfügung. ${ }^{6}$ Anonymus hat nicht nur die Verknüpfung der Ungarn mit den Hunnen vollbracht, er untermauert seine Behauptungen mit zusätzlichen Verbindungen zur Bibel (unter Einbeziehung der Figuren Gogs und Magogs), wodurch eine zeitlich und räumlich noch weiterführende Vorstellung über die Verwandtschaft und somit eine ,absolute“ Legitimation der Ungarn aufgestellt werden konnte:

5 Silagi 1991, S. 139, Anmerkung 22.

6 Szűcs 1992. 
Im Jahre des Herrn 819 war Ugek aus dem Geschlechte des Königs Magog der vornehmste Fürst von Skythien. Der nahm sich zum Weibe in Dentumoger die Tochter des eunedubelianischen Herzogs mit Namen Emesu. Mit ihr zeugte er einen Sohn, der den Namen Almus erhielt. Wegen eines göttlichen Wunders wurde er Almus genannt, denn seiner schwangeren Mutter war im Traum ein göttliches Gesicht in Gestalt eines Habichts erschienen, und es war, als käme er und schwängere sie. Und er verkündete ihr, aus ihrem Leibe würde ein Strom entspringen und ruhmreiche Könige aus ihren Lenden, aber nicht in ihrem eigenen Lande werde dies geschehen. [...]

Das tapfere und kriegsgewaltige Volk der Ungarn stammt also, wie wir sagten, vom skythischen Volke her, das sich in seiner eigenen Sprache Dentumoger nennt. Und jenes Land wurde zu voll von der Menge der darin erzeugten Menschen, so dass es ihnen weder Nahrung noch Raum genug geben konnte. Darum konnten jene sieben Fürsten, die Hetumoger bis auf den heutigen Tag heißen, die Enge des Landes nicht länger ertragen. Sie hielten Rat miteinander und beschlossen, den heimischen Boden zu verlassen und mit Waffengewalt ein Land zu erobern, darin sie wohnen könnten. Und sie nahmen sich vor, das Land Pannonien aufzusuchen, denn sie hatten vernommen, dies sei das Land König Ethelas, aus dessen Geschlecht Fürst Almus, Árpáds Vater, stammte. ${ }^{7}$

Der Autor komprimierte hier meisterhaft sehr unterschiedliche Elemente der ungarischen und europäischen Überlieferung. Elemente aus totemistischen Stammeslegenden, Strukturmerkmale der Steppenvölker, biblische Figuren und welthistorische Dimensionen sind in ein Geflecht kunstvoll zusammengefügt worden. Was allerdings in der Zeit des Anonymus in einem nicht von heutigen wissenschaftlichen Maßstäben geprägten Weltbild noch durchaus Stand halten konnte, ist nicht unverändert in unser heutiges Wissen zu integrieren.

Es muss hinzugefügt werden, dass eine weitere Überlieferung über die im Osten zurückgebliebenen Verwandten der Ungarn, die ebenso in den Gesta des Anonymus erwähnt wurde, ihre Spuren auch am königlichen Hofe hinterlassen hat, und es wurde mit der Suche nach diesen Verwandten im Osten im 13. Jahrhundert begonnen. ${ }^{8}$ Die viel versprechende Spur zu den früheren Siedlungsgebieten der Ungarn in der Wolga-Kama-Gegend, die im Bericht des Mönches Julianus erhalten geblieben ist, wurde mit dem Einfall der Mongolen endgültig verwischt. Reisende und Forscher der späteren Zeiten (z. B. Sándor Körösi Csoma, Antal Reguly oder Ármin Vámbéry) wurden dadurch gezwungen, andere, weiter entfernte Regionen (Indien, Sibirien bzw. die arabischen Länder) aufzusuchen, um noch ältere Spuren der Herkunft der Ungarn zu finden.

Eine ,frühwissenschaftliche“ Phase der Untersuchungen der Urgeschichte der Ungarn entsteht in der Spätrenaissance und in der darauf folgenden Reformationszeit, als die ungarische Sprache in den Mittelpunkt des Interesses rückt und

7 von Farkas 1955, S. 3-4.

8 Göckenjahn / Sweeney 1985, S. 67-125. 
die ersten, allerdings methodologisch sehr unvollkommenen und widersprüchlichen Theorien in Bezug auf die Verwandtschaft der ungarischen Sprache aufgestellt werden. Die Rolle der Sprache nimmt allgemein zu, erste, zunächst in den humanistischen Kreisen im Ausland aufgestellte Mutmaßungen werden artikuliert über die in Richtung finnougrische Völker zeigende Verwandtschaft der Sprache (Jugra) ${ }^{9}$, die im krassen Widerspruch zur historischen Überlieferung der Ungarn bezüglich ihrer Herkunft standen. Die rasche Entwicklung der Sprachwissenschaft, die in den weiteren Jahrhunderten ihre eigene, mit linguistischen Materialien und Mitteln massiv unterstützte Theorie über die Geschichte und Genealogie der ungarischen Sprache erstellen konnte, wurde zur schärfsten Konkurrentin der historischen Tradition. Tatsächlich ist die Herkunft der ungarischen Sprache heute wesentlich weiter und deutlicher geklärt als die Herkunft der ungarisch sprechenden Menschen. Dieser „Rückstand" der Geschichtswissenschaft hat im Übrigen damit zu tun, dass auch die wissenschaftlichen Disziplinen keine absolut mythologiefreien Gebilde sind, und die Traditionen bzw. die subjektiven und objektiven „Abhängigkeiten“ der gesellschaftsbezogenen Forschungsrichtungen können die Ausarbeitung bestimmter Ansätze innerhalb einer Disziplin erschweren, zeitweilig sogar verhindern.

Wie erfolgreich die Abkoppelung von den althergebrachten Traditionen durch die Staatsgründung gewesen ist, zeigen sehr charakteristisch unter anderem die verlorenen Schlachten gegen die Mongolen im 13. Jahrhundert, wo die Ungarn sozusagen von ihren eigenen, inzwischen aufgegebenen, traditionellen Kriegsführungsmethoden besiegt wurden, oder die anderthalb Jahrhunderte weilende teilweise Besatzung Ungarns durch die Osmanen. In diesem Falle, wo die Unterschiede des Glaubens (christliche Ungarn gegen die osmanischen Heiden) eine Zusammenarbeit mit den „Heiden“ nur aus taktischen Gründen, zur Erlangung der Souveränität des Ungarischen Königreichs, erlaubten, wird deutlich, wie tief der Wechsel in der Identität des ungarischen Volkes (vom einstigen, allerdings eher animistisch-schamanistisch geprägten Heiden zum Christen) vollzogen wurde. Trotz der oben erwähnten Kollaboration beharrt die ungarische Tradition in der Selbstdarstellung, ebenso wie zahlreiche andere in derselben Region dokumentierte Überlieferungen, auf dem als Wandermotiv zu bezeichnenden Titel Bollwerk des Christentums. In der ungarischen calvinistischen Tradition findet sogar die Anwendung einer biblischen Parallele statt: die Verluste und das Leid des Landes seien Strafen Gottes wegen der Sünden des Volkes gewesen, es wird also ein Bündnis mit Gott (das Motiv des ,auserwählten Volkes“) aufgestellt.

Kurz nach der Wiederherstellung der territorialen Einheit des Ungarischen Königreichs unter der Habsburger Krone (etwa nach 1686) wird die sprachliche Verwandtschaft mit dem weniger ruhmreichen Norden Europas (Lappen,

9 Fazekas 2001, S. 1144-1155. 
Finnen) in zwei Bahn brechenden Arbeiten nachgewiesen. ${ }^{10}$ Das Ende des 18., Anfang des 19. Jahrhunderts ist von einer lebhaften Diskussion gekennzeichnet, als diese ersten Versuche des wissenschaftlichen Sprachvergleichs die obige, von der historischen Tradition abweichende Behauptung in der Sprachverwandtschaft mit tatkräftiger internationaler Unterstützung der in Wien (der Sternwart, Maximilian Hell) und vor allem in Göttingen tätigen Gelehrten aufstellen. Pál Beregszászi Nagy, ein früher Vertreter der sprachverwandtschaftlichen Gedanken, fasste seine abweichende, weiterhin in Richtung Orient zeigende Meinung über die ungarische Sprache in einer groß angelegten Arbeit zusammen. Auffällig ist dabei, dass er das tat, obwohl er von diesem Vorhaben persönlich von August Schlözer in einem Brief gewarnt wurde. Schlözer, der Wegbereiter der später als historisch-vergleichend bezeichneten Methode in der Sprachwissenschaft, hat ihm folgendes mitgeteilt:

Türkisch, Persisch und Ungrisch sind verschieden an sich, wie Deutsch, Slawisch und Hebräisch: deswegen können doch im Ungrischen ein halbes Schock Worte vorkommen, die auch im Türkischen und Persischen sind. Wie viel Griechisches ist im Russischen, wie viel Lateinisches im Deutschen; und doch ist Russisch eine ganz andere Sprache wie Griechisch etc. Auch mit dem Hebräischen, Arabischen, Syrischen etc. hat das Ungrische keine Aehnlichkeit, als daß alle diese Sprachen Suffixa haben: hingegen mit dem Finnischen, oder Lappischen ist die Aehnlichkeit des Ungrischen frappant. ${ }^{11}$

Sajnovics und Gyarmathi haben das Selbstbild der Ungarn unmittelbar vor dem „nationalen Erwachen“ an einem äußerst empfindlichen Punkt getroffen. Dementsprechend hart waren die vehement ablehnenden öffentlichen Reaktionen auf die Entdeckung einer „nach Fisch riechenden Verwandtschaft“ und das Aufleben gegenteiliger Behauptungen. Auch die gebildeten Schichten, die Dichter haben sich an der Verspottung und Verurteilung der später sich als richtig erweisenden Theorie von Sajnovics beteiligt, wie zwei berühmt gewordene Gedichte illustrieren:

$\mathrm{Du}$, Sterngucker, ganz gleich wer du seist,

Kehre sofort zu deinen geliebten Verwandten heim,

Trockenfischbrei kannst du mit ihnen essen,

Sieh' jedoch ein: über unsere Sprache kannst du kein Urteil fällen.

(Lôrinc Orczy) $^{12}$

Hüten wir unser Volk vor Sajnovics' Joch:

Er leitet doch unsere Sprache aus Lappland her.

(Ábrahám Barcsay) ${ }^{13}$

10 Sajnovics 1770 und Gyarmathi 1799.

11 Beregszászi Nagy 1796, Seite V.

12 Erdődi 1970.

13 Ebd. 
Bemerkenswert ist, dass fast zur selben Zeit Immanuel Kant, ausgerechnet die von der ungarischen Öffentlichkeit so stark verpönten Verwandten der Ungarn zweimal als bedeutende Beispiele für die Anpassungsfähigkeit und Friedfertigkeit der Menschheit in seinem philosophischen Entwurf Zum ewigen Frieden erwähnt:

$\mathrm{Da} \beta$ in den kalten Wüsten am Eismeer noch das Moos wächst, welches das Rennthier unter dem Schnee hervorscharrt, um selbst die Nahrung, oder auch das Angespann des Ostjaken oder Samojeden zu sein [...] ist schon bewundernswürdig. [...]

Wir sehen nämlich Völker, die an der Einheit ihrer Sprache die Einheit ihrer Abstammung kennbar machen, wie die Samojeden am Eismeer einerseits, und ein Volk ähnlicher Sprache, zweihundert Meilen davon entfernt, im Altaischen Gebirge andererseits, wozwischen sich ein anderes, nämlich mongalisches, berittenes und hiermit kriegerisches Volk, gedrängt und so jenen Theil jenes Stammes weit von diesem, in die unwirthbarsten Eisgegenden, versprengt hat, wo sie gewiss nicht aus eigener Neigung sich hin verbreitet hätten[^);] - eben so die Finnen in der nordlichsten Gegend von Europa, Lappen genannt, von den jetzt eben so weit entfernten, aber der Sprache nach mit ihnen verwandten Ungern, durch dazwischen eingedrungene gothische und sarmatische Völker getrennt $[\ldots]^{14}$ [Hervorhebungen - T.F.]

Nicht einmal die Worte eines Kants haben - sofern sie überhaupt wahrgenommen wurden - in den vergangenen zweihundert Jahren die Einstellung des fachlich nicht ausgebildeten Publikums in Ungarn ändern vermocht. Entsprechend dem „Geist der Zeit“, konnten die erwähnten, mit „,deutschen“ (sprachvergleichenden) Methoden erzielten Erkenntnisse nur als Teil der Eroberung und Niederwerfung des nach Standesauffassung restriktiv auf den Adeligen definierten edlen ungarischen Volkes (wie z. B. auch die Modernisierung des Reiches bezweckenden Reformen vom Joseph II.) aufgefasst werden. Hieraus ist wiederum eine weitere, im öffentlichen Denken in Ungarn bis heute wirksame oppositionelle, auf die (orientalisch gefärbten) Ideen basierende Freiheitstradition entstanden, die alles Neue und alles, was der Überlieferung widerspricht, radikal ablehnt, weil sie darin nur ein Mittel zur Unterdrückung sieht und sofort aktualpolitische Gegenmaßnahmen fordert, denn nach dieser Vorstellung fällt eine um ihre ruhmreiche Vergangenheit beraubte Nation den (jeweils in unterschiedlichen Masken auftretenden, aber in den Absichten gleichen) kolonisierenden Bestrebungen leichter zum Opfer.

\section{Die Herausbildung der fiktiv-literarischen Herkunftstheorien}

Als eine erste Reaktion auf diese, gegen die Tradition sich richtende Behauptung, kann das Zeitalter des nationalen Erwachens angesehen werden, in dem

14 Kant 1795[1912], S. 363-5. 
die Romantik eine bunte, phantasievolle, mehr romanartige, als wissenschaftliche Forschung und Lehre gefordert hat. Diese „,fabulierende“ Wissenschaft hat einerseits Spuren in der Geschichtsforschung, also in der Entwicklung der Disziplin selbst hinterlassen, andererseits animierte sie gleichzeitig die Entstehung einer romantischen, historisierenden Epik, welche im Laufe der Zeit ganz wilde Vorstellungen, reine Fiktionen sogar in den wissenschaftlichen Diskurs über die Herkunft der Ungarn hat einbringen können! Nach 1800 sind über die Herkunft der Ungarn weitere, äußerst populäre, aber pseudowissenschaftliche Richtungen und zum Orient zeigende Theorien erschienen, wie z. B. die von István Horvát (1784-1846), der immerhin an der Universität von Pest seine Ansichten als Professor hat unterbreiten können und mit ihnen nachhaltige Popularität genoss. Pál Hunfalvy (1810-1891), ein Verfechter der zu dieser Zeit bereits anerkannten Methoden des sprachwissenschaftlichen Vergleichs, charakterisiert später Horváts Ansichten mit bissigen Worten:

Stephan Horvát schrieb mancherlei; 1825 gab er „Skizzen aus der ältesten Geschichte der ungarischen Nation“ (,Rajzolatok a magyar nemzet legrégibb történeteiből“) heraus. Die Scythen oder Ungarn kommen aus Nubien und Abyssinien; noch heute kann man auf der Landkarte Afrika's die Heimath der Magyaren, Kumanier, Petschenegen u. s. w. leicht auffinden. Nach Egypten gezogen, erbauten sie dort die Pyramiden und HORVÁT dankt der Vorsehung, dass es ihm gestattet worden, nach vielen tausend Jahren die Schrift der Parther - denn auch diese waren Magyaren - an einer egyptischen Mumie zu lesen. Herkules, Alexander der Große u. s. w. sind Vollblutmagyaren; überhaupt füllt diese Nation die ganze alte Geschichte aus; sie waren überall. Was in der hebräischen, in der finnischen Sprache an die magyarische erinnert, das kam aus dieser in jene. Auch in der Heiligen Schrift wimmelt es von magyarischen Namen und Magyarismen, aber nicht sowohl im Originaltexte, als vielmehr in der Vulgata des h. Hieronymus, der ein jazygischer Gelehrter (jász tudós) gewesen. Uebrigens ist ja auch der Psalmist David ein Székler und der Apostel Paulus ein Parther, also auch ein Ungar gewesen. - In solcher Weise führt Stephan Horvát mit vollem Ernste und grossem linguistischen und historischen Pathos fort, die Geschichte der Nation und Sprache zu skizzieren, und liefert ein kaum je erreichtes, aber gewiss nirgends übertroffenes Specimen gelehrter Verrücktheit. ${ }^{15}$

In dieser Phase spielten die Literatur und die allgemeine Bildung eine zunehmende Rolle beim „Wiedererobern“ und bei der Konzeptualisierung bestimmter fragmentarisch überlieferter epischer Stoffe und Motive der ungarischen Urgeschichte in die allgemeine kulturelle Überlieferung. Diese Änderung zeigt deutlich, dass parallel zu den wissenschaftlichen Entdeckungen und Fortschritten, die mythologischen Komponenten der Herkunftstradition der Ungarn in den Bereich der Fiktion ,umgesiedelt“ werden, und sie bleiben bis heute Elemente einer (z. T. konstruierten, z. T. rekonstruierten) epischen Tradition.

15 Hunfalvy 1877, S. 91-2. 
Die groß angelegten Versuche Mihály Vörösmartys (Zaláns Flucht, 1825) und vier Jahrzehnte später János Aranys (Budas Tod, 1863), um eine - wohl in dieser Form niemals existente - epische Tradition wieder zu beleben, bleiben jedoch Torsi. Die fertiggestellten Werkteile haben zwar den romantischen Erwartungen genüge getan, aber ihre Autoren waren allzu große Künstler, allzu empfindliche und kreative Dichter, um bei flachen historischen Tableaus zu bleiben: ihre Texte reflektieren ganz eindeutig die Probleme der Gattung, des Künstlers und die der ungarischen Gesellschaft in ihrer Zeit. Über Vörösmartys Epos können wir, im literaturgeschichtlichen Kontext festgestellt, lesen:

Am stärksten fühlt man jedoch in dem Epos einen lyrischen Oberton, der nicht bestimmt ist von dem Stolz über die Größe der heraufbeschworenen Zeit, sondern von der elegischen Trauer, die der Situation und der Stimmung des Dichters entspricht, der in die Vergangenheit zurückblickt. Die eindrucksvollsten und sich immer wiederholenden Bilder stellen die Nacht, den Tod, die Vergänglichkeit dar, und das Epos lässt auch die Erschütterung über das Schicksal des besiegten bulgarischen Fürsten Zalán deutlich werden. Durch die ,universale lyrische Anteilnahme“ gestaltet Vörösmarty das nationale Epos zu einer Universalität des menschlichen Schicksals, des Lebens und des Todes. ${ }^{16}$

Auch der spätere Versuch von Arany ist etwas Zeitgemäßes und somit völlig anderes geworden, als was die Anhänger der ruhmreichen Vergangenheit sich gewünscht hätten:

Budas Tod ist die Tragödie von Rolle und Persönlichkeit. König Buda teilt in einem Augenblick edler Aufwallung freiwillig den Thron mit seinem Bruder Attila. Damit übernehmen beide, Buda und Attila, eine Rolle, die nicht zu ihrem Wesen passt. Der eine ist zu schwach, der andere zu stark für eine geteilte Macht, und so müssen beide samt ihren Angehörigen zugrunde gehen. Zuerst stirbt Buda von Attilas Hand, dann Attila - das sollte in den weiteren Teilen der Trilogie dargestellt werden - infolge seiner Schuld. Doch außer der moralischen Ermahnung klingt aus dem wunderbar konzentrierten Werk auch noch eine andere Stimme heraus, oder vielleicht schwingt auch nur ein leiser Unterton mit. Vielleicht war es Absicht des Dichters, vielleicht nur eine Folge seiner Stilmittel, es ist der tragische Balladenton, eine aphoristisch düstere Stimmung, die sich aus der epigrammatischen Spannung ergibt, man hört das Grollen der Geschichte oder des Schicksals. Es ist nicht das Dröhnen der romantischen, geheimnisvoll-mythischen Historie mit den Sirenentönen des Verhängnisses, sondern ein in allen Details überblickbares, nüchtern kontrollierbares, fürchterliches Ineinandergreifen der Zahnräder, wie es in die Zeit des Positivismus passt. Man sieht wie die Räder einander antreiben und jeden, der in das Räderwerk gerät, zermalmen, nur den Zweck sieht man nicht. Es ist, wie gesagt, nur ein Unterton, zeugt aber davon,

16 Klaniczay 1977, S. 195. 
dass Aranys Illusionen über die historische Entwicklung seiner Zeit immer mehr dahinschwanden. ${ }^{17}$

Parallel zu den schönliterarischen Initiativen beginnt eine romantische Spurensuche auch nach der Volkstradition. ${ }^{18}$ Mór Jókai (1825-1904), der große romantische Erzähler Ungarns, sammelt während seiner zahlreichen Lesereisen im Land die lokalen Sagen und er plant diese - laut seiner gegen Ende seines Lebens geschriebenen Autobiographie - in einem epischen Großwerk miteinander zu verknüpfen:

Ich arbeite an einem ungarischen „Nibelungenlied“ bereits seit Jahren... Ich will die Geschichte der Staatsgründung von Árpád mit dichterischer Feder beschreiben, in welcher die Geschichte mit dem Sagenkreis verschmilzt. ${ }^{19}$ (Hervorhebung von mir, T. F.)

Aus diesem Werk wird zwar nichts, aber Jókai ist einer der Initiatoren, die an der überlieferten Stelle des ersten ungarischen „Landtages“, in (Puszta)Szer die Aufstellung eines um diese Zeit europaweit beliebten Unterhaltungs- und Bildungsmediums, eines monumentalen Panoramabildes, mit dem Thema Die ungarische Landnahme vorschlägt. Dieses Vorhaben wird $1896 \mathrm{zu}$ den Festlichkeiten des ungarischen Millenniums realisiert und kann - nach zahlreichen Unterbrechungen - auch heute besichtigt werden. Bezeichnend ist allerdings, dass die Festlichkeiten aus Anlass des tausendjährigen Jubiläums der ungarischen Landnahme fast in einem Rausch der türkeifreundlichen Stimmung stattgefunden haben. Diese Bewegung war eminent politisch gegen die auf dem Balkan sich anbahnende, von Russland dominierte panslawistische Bewegung gerichtet und bevorzugte eher die seit Jahrhunderten „,bekannten“ Gegner, die Türkei, als den russischen Zaren als „Patron“ in der Region. Im Mittelpunkt des Interesses und auch des Angebots an das schaulustige Publikum stand die romantisierte materielle Kultur der Ungarn, die kärgeren, problematischeren und andere Verbindungen aufzeichnenden Feststellungen der Wissenschaft wurden dabei nicht allzu stark berücksichtigt. Den romantischnationalen Grundgedanken dieser Vorstellungen hat am poetischsten der angesehene Literaturwissenschaftler Zsolt Beöthy (1848-1922) im Jahr des Millenniums so formuliert:

Aus der Dämmerung uralter Zeiten taucht vor unseren Augen die Gestalt eines Reiters empor, wie er auf der Wolga-Steppe ruhig dasteht und horcht. Seine Mütze spitz, sein Überwurf aus Pantherfell, sein sehniger Rumpf, als ob er mit dem kleinen Pferd zusammengewachsen wäre. Mit Adleraugen überblickt er die endlos scheinende Ebene, auf der jede Stelle scharf durch die glänzende Scheibe der Sonne beleuchtet wird. Er ist ruhig, hat keine Furcht und phantasiert nicht; nur das geht ihn an, was er sieht, und seine Augen, die sich an den Bildern der

17 Ebd., S. 251-52.

18 Ipolyi 1854.

19 Jókai 1898, S. 151. 
weiten Pussta, an dem scharfen Licht gestählt haben, sehen alles klar, was menschliche Augen von einer Stelle aus zu überblicken vermögen. Sein Köcher über die Schulter gehängt, das persische Schwert an der Seite: so lauert er auf den Feind. Wenn es nur einige sind, so nimmt er es mit ihnen auf, wenn sie in Scharen kommen, bringt er den Seinigen Nachricht. Für diese steht er jetzt Wache und ist zu allem entschlossen. Sein Blick erreicht auch die Ferne, die unabsehbar genannt wird: in einem entfernten schwarzen Punkt erkennt er den Geier, den schnellen, starken, grausamen Vogel seines Gottes. Das ist ein gutes Zeichen; er streichelt den Hals seines Pferdes und lässt seine Hand zuversichtlich auf dem Griff seines Schwertes ruhen. Er wartet auf die Zukunft und fühlt es, weiß es, dass die gemeinsame Sache auch seine Kraft brauchen wird. Seine Seele ist durch das Gefühl dieser Kraft und durch die Hingabe an seine Rasse erfüllt. ${ }^{20}$

In diese Stimmung passte nahezu ideal die Wiederbelebung der Herkunftsmythologie der einzigen ungarischen Bevölkerungsgruppe, der in Siebenbürgen lebenden Székler, die sich nicht als Árpáds Nachkommen bezeichnen, sondern sich direkt vom Hunnenkönig Attila, genauer gesagt von seinem jüngsten Sohn Csaba herleiteten. Die Tradition der ungarischen Kerbschrift, deren Zeugnisse hauptsächlich in den von den Széklern bewohnten Gebieten gefunden wurden, die anthropologischen Merkmale dieser Menschen haben auch in der Anthropologie und in der Geschichtswissenschaft verschiedene Theorien über ihre awarische bzw. kabarische Herkunft entstehen lassen. Elek Benedek (1859-1929) hat die Sagen, Mythen und Märchen der Székler gesammelt und die eigens bearbeiteten Fassungen der Texte in einem monumentalen fünfbändigen Werk ${ }^{21}$ veröffentlicht. Diese Publikation diente in der folgenden Zeit als Vorrat für Texte in verschiedenen Schulbüchern der allgemeinen Bildung: heute, wenn jemand nach der Ur- oder Vorgeschichte der Ungarn, nach ihren Wanderungen und historischen Helden fragt, bekommt er fast ausnahmslos die in Benedeks Buch stehenden Geschichten präsentiert.

Im Dezember 1905 läutete der epochale Dichter Endre Ady (1887-1919) den Sturm gegen das offizielle und verlogene Ungarnbild, gegen den falschen Mythos und die anachronistische Mentalität ein, er griff provokativ bereits mit dem Titel seines programmatischen Gedichtes auf die frühere, von dem calvinistischen Glauben stark geprägte biblische Herkunftstradition zurück:

Gog und Magogs Sohn

Gogs und Magogs Art entstamme ich,

Vergeblich stürm ich Tor und Mauern

Und dennoch hab ich euch befragt:

Darf man am Fusse der Karpathen trauern?

Durch Vereckes berühmten Pass ich kam,

Im Ohr noch Urmagyariens Sang mir schreit,

Ob ich bei Dévény wohl einbrechen darf,

20 zitiert in Keresztury [1999], S. 199-200.

21 Benedek 1894-96. 
Mit neuen Liedern einer neuen Zeit?

Giesst in die Ohren mir nur siedend Blei, Zum neuen Sänger Vazul macht mich so, Des Lebens neuen Liedern sei ich taub, Mit Füssen tretet mich, gemein und roh.

Bis dahin, weinend, qualvoll, nichts erwartend,

Steigt dennoch neubeschwingt das Lied, stolz fliegend,

Verflucht auch hundertmal von Pusztaszer,

Ist doch magyarisch es und neu und siegend. ${ }^{22}$

Ady stand mit seiner kritischen Ablehnung der romantischen Definitionen über die Nation, die verfälschte Tradition, die missbrauchte Kunst nicht allein. Die deheroisierenden, heute würde man sagen dekonstruierenden Züge seiner Dichtung um den Aufbau einer völlig neuen Kunst- und Weltauffassung sind bei anderen Künstlern und Gruppierungen am Anfang des 20. Jahrhunderts ebenso erkennbar: von Tivadar Csontváry Kosztkas provozierenden Skizzen zum Thema der Landnahme der Ungarn bis Béla Bartóks musikalischer Entwicklung von seiner frühen patriotischen Kossuth-Symphonie bis zu seinem späten „Allegro barbaro“ reichen die herausragenden Beispiele für diesen Wandel.

\section{Der Beitrag der letzten hundert Jahre}

Der erwähnte Anfang der „wissenschaftlichen“ Phase bedeutete den Anbruch einer bis heute anhaltenden Zeit der Konfrontationen zwischen den methodologisch fortgeschrittensten sprachwissenschaftlichen Feststellungen und den althergebrachten traditionellen Ansichten. Die wissenschaftliche Argumentation, die nach 1872 durch Hunfalvy und József Budenz (1836-1892) vertreten wurde, hat vor allem gegen die Behauptungen von Ármin Vámbéry (1832-1913) gekämpft. Diese Diskussion, der sogenannte ,ugrisch-türkische Krieg“" (ca. 1870-90) endete mit der wichtigen Feststellung, dass Sprache und Volk auch mit einander nicht absolut übereinstimmende Identitäten tragen können. Aber die Frage nach den Ursachen und Umständen dieser Diskrepanz zwischen den Zügen einer Sprache und ihrer Sprecher ist im Falle der Ungarn bis heute nicht befriedigend beantwortet worden. Eine in ihrer Struktur und ihrem Grundwortschatz eindeutig finnougrische Sprache wird von einem in seiner damaligen Kultur deutlich nach dem Muster der Turkvölker geprägten und in seiner Tradition sowie in seinen heute erkennbaren anthropologischen Merkmalen nachweisbar heterogenen Volk gesprochen.

22 Ady 1926, S. 6. 
Auf die Frage des Verhältnisses zwischen Sprache und Volk antwortete Gyula Szekfü (1883-1955), der führende Historiker der Zeit zwischen den Weltkriegen:

Die ebenen und leicht gewellten Länderstrecken vom Fuße der Karpathen bis zur Save und unteren Donau gerieten zu Ende des neunten Jahrhunderts in den Besitz des ungarischen Volkes. Wenn über die Urgeschichte desselben auch nicht in allen Einzelheiten Klarheit besteht, so darf heute doch mit Bestimmtheit angenommen werden, dass es aus der Vereinigung zweier verschiedener ethnischer Gruppen hervorgegangen ist. In den weiten Gebieten zwischen Ural und oberem Lauf der Wolga hat augenscheinlich einmal ein türkisch-tatarischer Hirtenstamm, also von Anlage und Beruf Eroberer und Herrenvolk, finnisch-ugrische Jäger und Fischer unterjocht. Die Sieger übernahmen die Sprache der Besiegten (Hervorhebung von mir, T. F.), und die gemeinsamen Schicksale vereinigten bald beide Gruppen zu einem Volke. Bereits im achten und neunten Jahrhundert finden wir die Ungarn als ethnisch einheitliches, festgeschmiedetes Nomadenvolk. Ihr Weg nach Westen führte von den Wolganiederungen bis zum Donaudelta, die nördlichen Gestade des Schwarzen Meeres entlang, deren Steppencharakter die Lebensweise eines berittenen Hirtenvolkes begünstigte. Die finnischen und türkischen Elemente sind zu dieser Zeit vollständig zusammengewachsen und werden von einer Einheitsorganisation umschlossen. Die sieben Geschlechtsverbände, die „Stämme“ des Volkes, zeigen bereits einen ausgeprägten ungarischen und nicht finnischen oder türkischen Charakter. Gegen Ende der Wanderzeit schloß sich ihnen ein achter Stamm an, die Kabaren, ein Zweig des türkisch-tatarischen Khasarenvolkes, wodurch das Verhältnis der finnischen und türkischen Elemente im ungarischen Volkstum noch mehr zugunsten der letzteren verschoben wurde. ${ }^{23}$

Nach 1920 und dem Gebietsverluste des historischen Ungarn, der in der Öffentlichkeit zum Teil als die Folge eines zu „,deutschtreuen“ Verhaltens in der k.u.k. Monarchie aufgefasst wurde, hat als Reaktion darauf eine hauptsächlich mit der Türkei sympathisierende öffentliche Meinung geherrscht, die zugleich außenpolitische Unterstützung genossen hat. Die frustrierte Phase zwischen den Weltkriegen brachte eine außenpolitische Neuorientierung und die wissenschaftlich-politische Unterstützung des damals populären, als ,turanisch“ bezeichneten Verwandtschaftsmythos in Ungarn mit sich, der Kreis der potentiellen Verwandten dehnte sich in Richtung China und Japan aus. Diese Entwicklung hat dann später weitere Folgen gezeigt, denn viele, erst nach dem Zweiten Weltkrieg und in der politischen Emigration außerhalb Ungarns vertretene eigenartige Verwandtschaftstheorien und Herkunftsmodelle der ungarischen Sprache lassen sich auf die phantasievollen Ideen der Zwischenkriegszeit zurückführen.

Nach dem Zweitem Weltkrieg wurde im Lande die sprachwissenschaftlich fundierte Herkunftstheorie - also der finnougrische Ursprung der ungarischen Sprache - von allen wissenschaftlichen Einrichtungen und Foren vertreten. Aufgrund der umfangreichen Forschungsarbeiten ist die Sprachwissenschaft am

23 Szekfü 1918, S. 12-13. 
deutlichsten imstande, über die Geschichte der ungarischen Sprache und dadurch über die zivilisatorisch-kulturelle und bedingt sogar über die geographische Vorgeschichte (Herkunft) der Ungarn verlässliche Aussagen zu machen. Davon abweichende Vorstellungen erhalten durch die Rollenverteilung unter der fremden (sowjetischen) Vorherrschaft einen politisch motivierten Rang als Opposition, d.h. alle hiergegen gerichteten (und auch alle nicht auf dem Niveau des Wissenschaftsbetriebes stehenden) Meinungen erhalten eine politisch motivierte Bedeutung (in der Emigration blüht die diesbezügliche „Literatur“ enorm auf), sie setzen einer Tradition des ,geistigen Freiheitskampfes der Nation" "fort. ${ }^{24}$

Nach 1989 wurden bei den Publikationen die wissenschaftlichen Maßstäbe stark herabgesetzt, die Öffentlichkeit wird unvermittelt mit (oft wieder ,aufgewärmten“), widersprüchlichen, ungeprüften, und oft haarsträubenden Theorien von verschiedenen Autoren konfrontiert (sprachliche Verwandtschaft mit dem Sumerischen, anthropologische mit den Japanern/Koreanern, kulturelle mit den Uiguren, die Aufstellung einer Theorie über die ganze Welt umfassende, über 20 tausend Jahre alte „Magya-Kultur“, etc., sowie die neulich entwickelten Ansichten aufgrund von angeblich festgestellten DNS-Merkmalen bei der Bevölkerung über die kosmische Herkunft der Ungarn von dem Stern Sirius). Auch bei den Geschichtswissenschaftlern kann das gesamte Spektrum der früheren Vorstellungen über die Herkunft der Ungarn wieder gefunden werden, sehr oft in deutlichem Widerspruch zu den Erkenntnissen der Sprachwissenschaft. Der Vorwurf des Verrats an der Nation und des Strebens nach wissenschaftlicher Hegemonie der finnougrischen Verwandtschaftstheorie wird auch in unseren Tagen immer wieder laut:

Was kann und kann etwas überhaupt der politische Inhalt, der Anlass einer Auffassung sein, in Bezug auf die Urgeschichte? Es kann sehr wohl sein, obwohl wir das nicht postulieren, dass die finnougrische Theorie von Anfang an in einem wissenschaftlichen Laboratorium zur Unterstützung einer politischen Auffassung geboren wurde. Sie ist lediglich auf die Habsburgischen Gesamtreichs-Bestrebungen gestoBen, die bemüht waren, die nationalen Besonderheiten zu vernichten, und sie hat sich zu deren wissenschaftlicher Untermauerung als geeignet erwiesen. Vor allem deshalb, weil die finnougrische Theorie behauptet, dass das Ungartum bloß mit einigen entfernten, zahlenmäßig kleinen und an der Peripherie der Weltereignisse lebenden Völkern verwandt ist, und diese Auffassung ist geeignet, im Kreise des in seinem nationalen Charakter sowieso zur Lethargie neigenden Ungartums das Gefühl der vereinsamten Verzagtheit zu stärken. Die des Selbstvertrauens, der Unterstützung der Brüdervölker beraubten Völker können dann leichter dem Willen des jeweiligen Eroberers unterworfen werden. Die politisch gestärkte finnougrische Theorie hat deshalb das Habsburgerreich überlebt und sie stellt bis in unsere Tage hin eine priviligierte Position dar, dank jener Bestrebungen, die kein Interesse an einer in ihrem Iden-

24 Rédei 1998. 
titäts- und Verwandtschaftsbewusstsein starken, selbstsicheren, aufsteigenden ungarischen Nation haben. ${ }^{25}$

(Hervorhebung von mir T. F.)

Neben den klassisch gefragten Disziplinen Sprachwissenschaft, Geschichte und Archäologie haben sich weitere Wissensbereiche der Forschung intensiv zugewandt. Die Ethnographie hat vor allem im Bereich der geistigen Folklore und in der Musik zahlreiche archaische Elemente systematisch entdeckt, deren Ursprung deutlich in Richtung Osten Europas, manchmal bis nach Asien hinein zeigt. Teilweise stimmen diese Phänomene mit vergleichbaren Merkmalen der Sprachverwandten überein, teilweise weichen sie von ihnen ab, vor allem aber sind diese Fragmente nicht als vollständiges System erhalten geblieben, d.h. ohne ein rekonstruierendes (manipuliertes) Konzept bleiben auch sie nur bedingt aussagekräftig.

In den letzten Jahren hat, mit Hilfe der Genomforschung, die Anthropologie auf sich aufmerksam gemacht. Ihre neuesten Versuche bezüglich der $\mathrm{Ab}$ stammung der Ungarn und ihrer Kultur zeigen in eine ,alt-neue“ Richtung, nach dem Nordwesten des heutigen China. Jene Ansichten und Funde, die in diese Konzeption passen, wurden zuletzt in einem kontroversen Buch von István Kiszely zusammengefasst. ${ }^{26}$

\section{Schlussbemerkungen}

Grob betrachtet gab es mehrere, von einander deutlich zu trennende Phasen in der ungarischen Kulturgeschichte, in denen die variierenden Mythen einer orientalischen Abstammung des Volkes stärker vertreten wurden. Nach der Staatsgründung, zur Zeit des nationalen Erwachens, in der Ära der Romantik, beim tausendjährigen Jubiläum der Landnahme und fast immer, wenn das Land seine Integrität an fremde Mächte abtreten musste, wurden die charakteristischen Motive der orientalischen, geheimnisumwitterten und ruhmreichen Urgeschichte der Ungarn wieder belebt. Die Suche nach Verwandten, nach einer (sicheren) Urheimat, nach Ruhm in der Vergangenheit wurde in kritischen Situationen, bei gravierenden Herausforderungen immer stärker, sie ist ein - psychologisch durchaus verständlicher - Abwehrmechanismus, damit nicht die wahrhaftigen Probleme in den Vordergrund rücken, die die tatsächlichen, aktuell vorhandenen Tugenden auf den Prüfstand stellen würden. Historische Änderungen, welche das Gefühl eines Identitätsverlustes unter erzwungenen Bedingungen erzeugen können (teilweise wird heute der EU-Beitritt so verstanden), sind ganz besonders geeignet, immer wieder in tatsächliches oder zumindest mentales Opponieren, in Rebellionen, in den Zusammenstoß von integrierenden und separatistischen Traditionen zu münden.

25 Pusztaszeri ca. 1997, S. 8.

26 Kiszely 1996. 
Die zahlreichen Niederlagen der ungarischen Geschichte des 16.-20. Jahrhunderts werden bis heute häufig auf das „Verkommen“ der einst so großen ungarischen Nation zurückgeführt, und an diesen einstigen Ruhm wird krampfhaft erinnert, damit die Kleinheit der Gegenwart und das häufige Versagen der Betroffenen nicht ins Auge fallen. ${ }^{27}$

\section{Literaturverzeichnis}

Ady, Andreas, 1926: Auf dem Flammenwagen der Lieder. (Übertragen von Albert Hetényi-Heidberg), Budapest.

Benedek, Elek, 1894-96: Magyar mese- és mondavilág [Ungarische Märchen- und Sagenwelt] I-V. Budapest.

Beregszászi Nagy, Pál, 1796: Ueber die Aehnlichkeit der hungarischen Sprache mit den morgenlaendischen. Leipzig, Breitkopf und Haertel.

Erdődi, József, 1970: „Sajnovics, der Mensch und Gelehrte“, in: Acta Linguistica Academiae Scientiarum Hungaricae 20 (1970), 3-4, 291-322.

Farkas, Julius von (Hrsg.), 1955: Ungarns Geschichte und Kultur in Dokumenten. Wiesbaden.

Fazekas, Tiborc, 2001: „Die Entdeckung der Verwandtschaft der finnougrischen Sprachen", in: History of Language Sciences/Geschichte der Sprachwissenschaften, Band II. Berlin, New York, 1144-1155.

Göckenjahn, Hansgerd / Sweeney, James R. (Hrsg.), 1985: Der Mongolensturm. Graz, Wien, Köln.

Gyarmathi, Sámuel, 1799: Affinitas... Göttingen.

Heiszler, Vilmos: Romantikus leszármazási elméletek és nemzeti önszemlélet [Romantische Herkunftstheorien und nationale Selbstbetrachtung]. http://www.civic-edu.net/ inner.php?lang $=$ hu

Hunfalvy, Paul, 1877: „Die ungarische Sprachwissenschaft - Historischer Überblick“, in: Literarische Berichte aus Ungarn. Budapest.

Ipolyi, Arnold, 1854: Magyar Mythologia [Ungarische Mythologie]. Pest.

Jókai, Mór, 1898: „Önéletírásom“ [Meine Autobiographie], in: Jókai Mór összes munkái [Sämtliche Werke von Mór Jókai]. Budapest. (Übersetzung: Tiborc Fazekas)

Kant, Immanuel, 1795: Zum ewigen Frieden, in: Kant's gesammelte Schriften. Band 8. Berlin, 1912.

Kellner, Maximilian Georg, 1997: Die Ungarneinfälle im Bild der Quellen bis 1150. München.

Keresztury, Dezső, 1999: Menschen, Werke, Verbindungen. o. O. Pécs.

Kiszely, István, 1996: A magyarság őstörténete [Die Urgeschichte des Ungartums] I-II. Budapest.

Klaniczay, Tibor, (Hrsg.) 1977: Handbuch der ungarischen Literatur. Budapest.

27 Siehe dazu: Heiszler: http://www.civic-edu.net/inner.php?lang=hu 
Kovács, László / Veszprémy, László (Hrsg.), 1996: A honfoglaláskor írott forrásai [Die schriftlichen Quellen der Zeit der Landnahme]. Budapest.

Pusztaszeri, László, ca. 1997: Az élő Árpádok [Die lebendigen Árpáden]. (Übersetzung: Tiborc Fazekas). Eger, o. J.

Rédei, Károly, 1998: „Östörténetünk kérdései“ [Fragen über unsere Urgeschichte], in: Budapesti finnugor füzetek [Finnougristische Hefte aus Budapest] 10. Budapest.

Róna-Tas, András, 1999: Hungarians and Europe in the Early Middle Ages. Budapest.

Sajnovics, János, 1770: Demonstratio... Tyrnavia (Nagyszombat, heute Trnava in der Slowakei).

Silagi, Gabriel (Hrsg.) (unter Mitarbeit von Veszprémy, László), 1991: Die „Gesta Hungarorum " des anonymen Notars. Sigmaringen.

Szekfü, Julius [Gyula] 1918: Der Staat Ungarn. Stuttgart und Berlin.

Szűcs, Jenő, 1992: A magyar nemzeti tudat kialakulása [Die Herausbildung des ungarischen Natinalbewußtseins]. Szeged. 